\section{Diferentes momentos de aplicação de gonadotrofina coriônica equina em protocolo de inseminação artificial em tempo fixo para vacas de leite}

[Different times of application of equine chorionic gonadotropin in fixed-time artificial insemination protocols for dairy cows]

\section{M.P. Lents}

https://orcid.org/0000-0002-0434-4017 E.E.G. Pinheiro

https://orcid.org/0000-0002-6302-2085 C.S. França

https://orcid.org/0000-0003-0595-1246 R.D.L. Jesus

https://orcid.org/0000-0002-4380-5218

\author{
M.L. Araújo ${ }^{1}$, L.P. Barbosa ${ }^{1}$, C.E.A. Biscarde ${ }^{2}$, C.S. Mendes ${ }^{1}$, M.P. Lents ${ }^{1}$, \\ E.E.G. Pinheiro ${ }^{2}$, C.S. França ${ }^{1}$, R.D.L. Jesus ${ }^{1}$
}

${ }^{1}$ Universidade Federal do Recôncavo da Bahia - Cruz das Almas, BA

${ }^{2}$ Universidade Federal da Bahia - Salvador, BA

\begin{abstract}
RESUMO
Avaliaram-se diferentes momentos de aplicação da gonadotrofina coriônica equina (eCG) em protocolos de inseminação artificial em tempo fixo (IATF) para vacas de leite. Foram utilizadas 76 fêmeas, as quais receberam, no dia zero (D0) do protocolo, dispositivos intravaginais de progesterona, sendo esses retirados no D9, e os animais foram, então, distribuídos aleatoriamente em três tratamentos: T1 aplicação de eCG no momento da retirada dos dispositivos; T2 e T3 - aplicação de eCG 48h e 24h antes da retirada dos dispositivos, respectivamente. No D10 os animais receberam $1 \mathrm{mg}$ de GnRH, e a IATF foi realizada 52 horas após a retirada do implante. Não houve diferença $(\mathrm{P}>0,05)$ para intervalo entre a retirada do implante à ovulação $(72,56 \pm 3,92 \mathrm{~h})$, o diâmetro do maior folículo no $\mathrm{D} 9(10,88 \pm 1,49 \mathrm{~mm})$, o diâmetro do folículo ovulatório $(15,15 \pm 1,16 \mathrm{~mm})$ e do segundo maior folículo $(7,49 \pm 0,52 \mathrm{~mm})$, a taxa de crescimento folicular $(1,38 \pm 0,04 \mathrm{~mm} / \mathrm{dia})$, a taxa de ovulação $(96,67 \%)$, o intervalo entre diâmetro final e inicial do folículo dominante $(73,49 \pm 3,84 \mathrm{~h})$, a área de corpo lúteo $\left(2,27 \pm 0,43 \mathrm{~cm}^{2}\right)$, a porcentagem de CL no ovário direito $(53,00 \%)$ e no esquerdo $(26,33 \%)$ e a taxa de gestação $(33,33 \%)$. O momento da aplicação da eCG não influenciou na eficiência do protocolo. Recomenda-se a utilização da eCG no momento da retirada do implante por otimização do manejo.
\end{abstract}

Palavras-chave: inseminação artificial, reprodução, sincronização da ovulação

\begin{abstract}
Different times of application of equine chorionic gonadotropin $(e C G)$ in Fixed-Time Artificial Insemination (FTAI) protocols for dairy cows were evaluated. A total of 76 females were used, which received intravaginal progesterone devices on day zero $(D 0)$ of the protocol, which were withdrawn on $D 9$, and the animals were then randomly distributed in three treatments: T1-application of eCG at the time of device withdrawal; $T 2$ and T3 - application of eCG $48 \mathrm{~h}$ and $24 \mathrm{~h}$ before withdrawal of the devices, respectively. On D10 the animals received $1 \mathrm{mg}$ of GnRH and the FTAI was carried out 52 hours after the removal of the implant. There was no difference $(P>0.05)$ for interval from implant removal to ovulation $(72.56 \pm 3.92 \mathrm{~h})$, diameter of the largest follicle of $D 9(10.88 \pm 1.49 \mathrm{~mm})$, diameters of ovulatory follicle $(15.15 \pm 1.16 \mathrm{~mm})$ and of the second largest follicle $(7.49 \pm 0.52 \mathrm{~mm})$, follicle growth rate $(1.38 \pm 0.04 \mathrm{~mm} /$ day), ovulation rate $(96.67 \%)$, interval between final diameter and initial diameter of dominant follicle $(73.49 \pm 3.84 \mathrm{~h})$, corpus luteum area $\left(2.27 \pm 0.43 \mathrm{~cm}^{2}\right)$, percentage of $C L$ in right ovary (53.00\%) and in left ovary (26.33\%) and pregnancy rate (33.33\%). The time of the application of eCG did not influence the efficiency of the protocol. We recommend the use of eCG at the time of the removal of the implant for management optimization.
\end{abstract}

Keywords: artificial insemination, reproduction, ovulation synchronization

Recebido em 13 de setembro de 2017

Aceito em 15 de abril de 2019

E-mail: monnalopes@hotmail.com 


\section{INTRODUÇÃO}

Entre as biotecnologias utilizadas na reprodução animal, a inseminação artificial (IA) é uma importante ferramenta para acelerar o melhoramento genético (Vishwanath, 2003), contudo a detecção de estro é o principal obstáculo para a utilização da IA em bovinos, que exige tempo e mão de obra especializada. Além disso, a curta duração do comportamento estral de aproximadamente $11 \mathrm{~h}$ e a alta incidência de estro à noite, variando de 30 a $50 \%$, tornam mais custosa a detecção de estro, dificultando a utilização dos programas convencionais de IA (Pinheiro et al., 1998).

Os estudos sobre dinâmica folicular ovariana em bovinos (Pierson e Ginther, 1988; Sirois et al., 1988) possibilitaram o emprego de tratamentos hormonais que controlam o crescimento folicular ovariano e a sincronização da ovulação, permitindo o uso de inseminação artificial em tempo fixo (IATF). Marques et al. (2003), ao trabalharem com vacas mestiças Bos taurus indicus primíparas pós-parto, mostraram que o tratamento com gonadotrofina coriônica equina (eCG) aumentou as concentrações plasmáticas de progesterona $\left(\mathrm{P}_{4}\right)$ aos 12 dias após o término do tratamento e obtiveram $76 \%$ de ovulação pósIATF+eCG, sem aumentar significativamente o diâmetro do folículo ovulatório dominante e a área do corpo lúteo. Esses dados indicaram que o tratamento com eCG aumentou a produção de $\mathrm{P}_{4}$ pelo corpo lúteo $(\mathrm{CL})$ e pode ser uma alternativa para melhorar o desempenho reprodutivo de vacas em anestro pós-parto, submetidas à IATF.

A eCG estimula a produção de estradiol por meio das células da granulosa e da $\mathrm{P}_{4}$ pelo CL (Baruselli et al., 2004). O aumento na produção de estradiol folicular induzido pela eCG parece estar ligado a um aumento na produção de RNAm para citocromo P450 e receptores de hormônio luteinizante (LH) (Soumano et al., 1998). Considerando o tempo de meia-vida e a afinidade para os receptores de hormônio folículo estimulante (FSH) e LH (Murphy e Martinuk, 1991), a administração de eCG dois dias antes de retirada da fonte exógena de $\mathrm{P}_{4}$ pode ser uma estratégia para aumentar o tamanho do folículo dominante no momento da ovulação em bovinos submetidos a protocolos de IATF (Tortorella et al., 2013).
Tortorella et al. (2013) sugerem que o tamanho do folículo dominante foi maior quando a eCG foi administrada um dia antes da retirada do CIDR, com uma taxa de ovulação superior à dos protocolos padrão. Esse achado demonstra a possibilidade de se investigarem novos protocolos com diferentes momentos de aplicação da eCG, a fim de se certificar qual melhor momento terá melhor eficiência aos protocolos de inseminação artificial em tempo fixo (IATF) para vacas. Por essas razões, o presente estudo teve como objetivo avaliar diferentes momentos de aplicação da eCG em protocolos de IATF para vacas de leite.

\section{MATERIAL E MÉTODOS}

Foram realizadas duas etapas experimentais aprovadas pelo Comitê de Ética no Uso de Animais (Ceua) da Universidade Federal da Bahia (UFBA), protocolado sob número 342015. A primeira etapa experimental foi conduzida no Setor de Bovinocultura de Leite da Universidade Federal do Recôncavo da Bahia - UFRB, no campus de Cruz das Almas, Bahia, localizado a 12³9'54.9” de latitude sul, 3904'36.9” de longitude oeste, com altitude de $195 \mathrm{~m}$ acima do nível do mar, no período de junho a agosto, compreendendo o período de inverno (Tabela, 2013; Estações, 2015).

Foram selecionadas 39 vacas adultas, mestiças, por avaliação ginecológica com ultrassonografia (Pie Medical, modelo AquilaVet ${ }^{\circledR}$, transdutor linear de $6 \mathrm{MHz})$, com escore de condição corporal de 3,56 $\pm 0,6$, segundo Edmonson et al. (1989). Os animais foram mantidos em regime extensivo de criação, em pasto de aruana (Panicum maximum cv. Aruana), com água e sal mineral ad libitum.

As vacas foram distribuídas aleatoriamente em três tratamentos (T) e as aplicações hormonais foram realizadas por via intramuscular no membro posterior. No dia zero (D0) do protocolo, todas as vacas receberam dispositivo intravaginal impregnado com progestágenos (Primer $^{\circledR}$, Tecnopec, Brasil) e $3 \mathrm{mg}$ de benzoato de estradiol (Estrogin ${ }^{\circledR}$, Biofarme, Brasil). No D9 do protocolo, foram retirados os dispositivos e os animais foram distribuídos nos tratamentos, sendo: T1 $(\mathrm{n}=13)$ : aplicação de $150 \mu \mathrm{g}$ de um análogo sintético da $\mathrm{PGF}_{2} \alpha$, o d-cloprostenol (Prolise $^{\circledR}$, Arsa, Argentina) e 400UI de eCG 
(Novormon ${ }^{\circledR}$, Syntex, Argentina) no momento da retirada do dispositivo; T2 $(n=13)$ : aplicação de $150 \mu \mathrm{g}$ de d-cloprostenol e 400UI de eCG aplicada $48 \mathrm{~h}$ antes da retirada do dispositivo; e T3 (n=13): aplicação de $150 \mu \mathrm{g}$ de d-cloprostenol e 400UI de eCG, aplicada $24 \mathrm{~h}$ antes da retirada do dispositivo. No D10 do protocolo, todos os animais receberam 1mg de GnRH (Gestran Plus, Tecnopec $^{\circledR}$, Brasil) e a IATF foi realizada $52 \mathrm{~h}$ após a retirada do implante. O sêmen utilizado na inseminação foi proveniente de banco de sêmen de centrais de congelamento certificadas, sendo utilizada uma palheta de $0,25 \mathrm{~mL}$ por fêmea.

Após a retirada do implante, 10 animais de cada tratamento foram monitorados para determinação do momento da ovulação, por meio de avaliações ultrassonográficas por via transretal, a cada 24 horas, até 24 h após a detecção da ovulação, para se determinar o intervalo entre a retirada do implante à ovulação (h); o tamanho do maior folículo do dia nove do protocolo $(\mathrm{mm})$; o tamanho do folículo ovulatório (mm); o tamanho do segundo maior folículo ( $\mathrm{mm})$; a taxa de crescimento folicular $(\mathrm{mm} / \mathrm{d})$; e o intervalo entre o diâmetro final e o inicial folicular (h). As imagens dos ovários foram obtidas por ultrassonografia via transretal (Pie Medical, modelo AquilaVet ${ }^{\circledR}$, transdutor linear de 6,0MHz).

A avaliação do corpo lúteo (CL) foi realizada nove dias após a retirada do dispositivo intravaginal, por meio de avaliação ultrassonográfica, para determinação da área de corpo lúteo $\left(\mathrm{cm}^{2}\right)$ e porcentagem de corpo lúteo no ovário direito e no esquerdo $(\%)$.

A segunda etapa experimental foi realizada na Fazenda JF Reunidas, no distrito de Tanquinho, Bahia, localizado a $11^{\circ} 58^{\prime} 43^{\prime}$ de latitude sul, $39^{\circ} 06^{\prime} 14$ de longitude oeste, altitude de $248 \mathrm{~m}$ acima do nível do mar, que apresenta clima tropical quente úmido, compreendendo os meses de julho a agosto, período de inverno Estações, 2015).

Foram utilizadas 37 fêmeas adultas mestiças da raça Girolando, previamente selecionadas por meio de avaliação ginecológica com ultrassonografia (Pie Medical, modelo ÀquilaVet ${ }^{\circledR}$, transdutor linear de $6 \mathrm{MHz}$ ), com escore de condição corporal de $3,45 \pm 0,31$, segundo Edmonson et al. (1989). Os animais foram mantidos em regime extensivo de criação, em pasto de aruana (Panicum maximum cv. Aruana), com água e sal mineral ad libitum.

As vacas foram distribuídas aleatoriamente em três tratamentos $(\mathrm{T})$, e o protocolo hormonal foi realizado igualmente à primeira etapa, respeitando o protocolo correspondente a cada tratamento, sendo T1 $(\mathrm{n}=12) ; \mathrm{T} 2 \quad(\mathrm{n}=13) ; \mathrm{T} 3$ $(n=12)$. Foram avaliados, nessa etapa experimental, os parâmetros do corpo lúteo (CL) e a taxa de gestação. A avaliação do CL foi realizada nove dias após a retirada do dispositivo intravaginal, por meio de avaliação ultrassonográfica. O diagnóstico de gestação foi realizado 30 dias após a IATF, mediante ultrassonografia. As imagens dos ovários e úteros foram obtidas por ultrassonografia via transretal (Pie Medical, modelo ÀquilaVet ${ }^{\circledR}$, transdutor linear de $6,0 \mathrm{MHz}$ ).

Para ambas as etapas experimentais, foi utilizado o delineamento inteiramente ao acaso (DIC) e avaliado o efeito fazenda entre os dados das duas etapas experimentais. Os dados foram inicialmente avaliados quanto à normalidade por meio do teste de Shapiro-Wilk. As variáveis que apresentaram distribuição normal foram analisadas por ANOVA a $5 \%$ de probabilidade e as variáveis não paramétricas foram analisadas por meio do teste de Kruskal-Wallis (SPSS, versão 21 (1989-2012)).

\section{RESULTADOS E DISCUSSÃO}

Não foi observado efeito fazenda entre os dados das duas etapas experimentais, dessa forma foram avaliados conjuntamente. Kiya et al. (2014), ao trabalharem com ovinos submetidos a protocolos de sincronização para IATF, com aplicação de eCG, $24 \mathrm{~h}$ ou $48 \mathrm{~h}$ antes da retirada da fonte de progesterona, também não obtiveram diferença entre seus tratamentos, com $52,50 \pm 7,55 \mathrm{~h}$ e $51,00 \pm 6,00 \mathrm{~h}$, para intervalo da retirada da fonte de progesterona à ovulação; $6,60 \pm 0,35 \mathrm{~mm}$ e $8,57 \pm 2,47 \mathrm{~mm}$, para tamanho do maior folículo e $0,65 \pm 0,30 \mathrm{~mm} /$ dia e $1,25 \pm 0,63 \mathrm{~mm} / \mathrm{dia}$, na taxa de crescimento folicular, para aplicação 24 e $48 \mathrm{~h}$ antes da remoção do dispositivo de progesterona, respectivamente. 
Diferentes momentos de...

Tabela 1. Crescimento folicular e momento de ovulação de vacas de leite submetidas a protocolos de sincronização de ovulação utilizando diferentes momentos da aplicação da gonadotrofina coriônica equina

\begin{tabular}{|c|c|c|c|c|}
\hline Parâmetros & T1 (controle) & $\mathrm{T} 2$ (48h antes) & T3 (24h antes) & Média total \\
\hline IRO $(h)^{1}$ & $77,05 \pm 10,06$ & $70,81 \pm 11,58$ & $69,83 \pm 0,95$ & $72,56 \pm 3,92$ \\
\hline TMFD9 $(\mathrm{mm})^{1}$ & $9,17 \pm 2,41$ & $11,88 \pm 2,82$ & $11,58 \pm 2,46$ & $10,88 \pm 1,49$ \\
\hline $\mathrm{TMF}(\mathrm{mm})^{1}$ & $13,82 \pm 2,11$ & $15,95 \pm 2,40$ & $15,67 \pm 2,02$ & $15,15 \pm 1,16$ \\
\hline $\operatorname{TSMF}(\mathrm{mm})^{1}$ & $8,07 \pm 1,54$ & $7,35 \pm 1,94$ & $7,05 \pm 1,54$ & $7,49 \pm 0,52$ \\
\hline $\mathrm{TCF}(\mathrm{mm} / \mathrm{d})^{1}$ & $1,40 \pm 0,07$ & $1,32 \pm 0,04$ & $1,40 \pm 0,06$ & $1,38 \pm 0,04$ \\
\hline Taxa ovulação $(\%)^{2}$ & $10 / 10(100 \%)$ & $10 / 10(100 \%)$ & $9 / 10(90 \%)$ & $96,67 \pm 5,77$ \\
\hline IDFDI $(h)^{1}$ & $77,47 \pm 9,76$ & $73,18 \pm 7,27$ & $69,81 \pm 0,92$ & $73,49 \pm 3,84$ \\
\hline
\end{tabular}

T1 (n=10); T2 (n=10); T3 (n=10); IRO=intervalo da retirada à ovulação, TMFD9=tamanho do maior folículo do dia 9 do protocolo, TMF=tamanho do folículo ovulatório, TSMF=tamanho do segundo maior folículo, TCF=taxa de crescimento folicular, IDFDI= intervalo entre diâmetro final menos inicial. Não houve diferença entre os tratamentos por meio de ${ }^{1}$ ANOVA $(\mathrm{P}>0,05) \mathrm{e}^{2}$ Kruskal Wallis $(\mathrm{P}>0,05)$.

Em contraposição, Ali (2007) observou que a antecipação da eCG resultou na redução precoce do número de folículos menores, acompanhada pelo aparecimento de folículos médios e grandes, possibilitando melhores taxas de ovulação e fecundação. O tamanho do folículo no dia nove do protocolo, correspondente ao momento da retirada do implante, foi de $9,17 \pm 2,41(\mathrm{~T} 1)$; $11,88 \pm 2,82 \quad$ (T2); 11,58 $\pm 2,46 \quad$ (T3) $\quad(\mathrm{P}>0,05)$ (Tab. 1). Apesar de não ter havido diferença entre os tratamentos neste estudo, pesquisas comprovam que o mecanismo pelo qual a eCG pode ser considerada eficiente nos protocolos de sincronização pode ser justificado por um aumento da taxa de crescimento folicular, levando o folículo à categoria de folículos médios e diminuindo a ocorrência de atresia natural desses folículos, melhorando o seu crescimento (Bister et al., 1999; Mandiki et al., 2000).

Entre as particularidades fisiológicas que diferenciam vacas Bos indicus de Bos taurus, pode-se citar o diâmetro folicular na divergência e o diâmetro no qual o folículo atinge capacidade ovulatória, sendo o diâmetro folicular, em fêmeas Bos indicus, inferior $(10 \mathrm{~mm})$ ao de fêmeas Bos taurus (12mm) (Gimenes et al., 2008), como observado no presente estudo para o valor médio do folículo pré-ovulatório encontrado de 15,14 $\pm 1,15 \mathrm{~mm}$ (Tab. 1).

O tamanho médio do segundo maior folículo foi de $7,49 \pm 0,52 \mathrm{~mm}$ e a taxa de crescimento folicular média entre os tratamentos foi de 1,38 $\pm 0,04 \mathrm{~mm} /$ dia; $(\mathrm{P}>0,05)$ (Tab. 1). Ali (2007) observou que a antecipação da eCG resultou na redução precoce do número de folículos menores, acompanhada pelo aparecimento de folículos médios e grandes, possibilitando melhores taxas de ovulação e fecundação, o que mostra a importância da utilização da eCG dentro dos protocolos de sincronização, a fim de melhorar sua eficiência.

Ali (2007) utilizou eCG dois dias antes da retirada da esponja em ovelhas e obteve maior taxa de ovulação $(\mathrm{P}<0,05)$ no grupo com aplicação antecipada de eCG comparado ao controle. Esse resultado diverge do encontrado no presente trabalho, que não apresentou diferença $(\mathrm{P}>0,05)$ (Tab. 1) para taxa de ovulação (10/10; 10/10; 9/10), para T1, T2 e T3, respectivamente, com média $96,66 \%$ entre os tratamentos. No entanto, Tortorelle et al. (2013), ao trabalharem com vacas de leite, comparando a aplicação de eCG $48 \mathrm{~h}$ antes da retirada do implante (G1) com um grupo que recebeu eCG no momento da retirada (G2), obtiveram taxa de ovulação maior para o G1 (6/7) do que para o G2 $(3 / 6)$.

Não houve diferença $(\mathrm{P}>0,05)$ para os valores encontrados de área de corpo lúteo e taxa de gestação entre os tratamentos (Tab. 2). Sartori et al. (2002) relataram uma correlação positiva entre o CL e o volume de produção de $\mathrm{P}_{4}$ em sete dias após a ovulação em novilhas e vacas leiteiras. No entanto, a correlação entre a $\mathrm{P}_{4}$ sérica e o tamanho do CL parece existir somente durante a formação inicial do CL (Mann, 2009). Tendo em vista esse conhecimento, o protocolo de IATF descrito por Tortorelle et al. (2013), com aplicação da eCG $48 \mathrm{~h}$ antes da retirada do implante de $\mathrm{P}_{4}$, foi projetado com o intuito de aumentar o tamanho do folículo pré-ovulatório e 
consequentemente o volume do $\mathrm{CL}$ inicial, aumentando, assim, a concentrações de $\mathrm{P}_{4}$ após a ovulação. Entretanto, os resultados de área de CL do presente estudo não diferiram entre si, com valor médio entre os tratamentos de $2,27 \pm 0,43 \mathrm{~cm}^{2}$ (Tab. 2), não corroborando a hipótese levantada por Tortorelle et al. (2013).

Vianna et al. (1999), ao estudarem animais da raça Gir, encontraram $57,14 \%$ de incidência de CL no ovário direito, no entanto Neves e
Marques Júnior (2006), ao analisarem ovários de vacas Nelore, encontraram maior incidência do corpo lúteo no ovário esquerdo, o que mostra uma diferença da frequência da incidência de CL entre as raças. Esses dados corroboram os achados do presente trabalho, que obtiveram um índice numérico de CL no ovário esquerdo maior que no ovário direito (Tab. 2), levando em consideração que os animais estudados foram meio-sangue Girolando.

Tabela 2. Área de corpo lúteo e diagnóstico de gestação de vacas de leite submetidas a protocolo de sincronização de ovulação utilizando diferentes momentos de ampliação da gonadotrofina coriônica equina

\begin{tabular}{lcccc}
\hline Parâmetros & T1 (controle) & T2 (48h antes) & T3 (24h antes) & Média total \\
\hline ACL $\left(\mathrm{cm}^{2}\right)^{1}$ & $1,78 \pm 1,19$ & $2,44 \pm 1,20$ & $2,58 \pm 1,42$ & $2,27 \pm 0,43$ \\
CLOD $(\%)^{2}$ & $10 / 22(45 \%)$ & $14 / 23(60 \%)$ & $12 / 22(54 \%)$ & $53,00 \pm 7,55$ \\
CLOE $(\%)^{2}$ & $7 / 22(31 \%)$ & $5 / 23(21 \%)$ & $6 / 22(27 \%)$ & $26,33 \pm 5,03$ \\
DG $(\%)^{2}$ & $10 / 25(40 \%)$ & $6 / 26(24 \%)$ & $9 / 25(36 \%)$ & $33,33 \pm 8,33$ \\
\hline
\end{tabular}

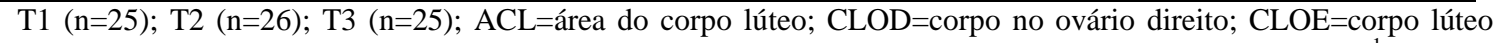
ovário esquerdo; DG=diagnóstico de gestação. Não houve diferença entre os tratamentos por meio de ${ }^{1}$ ANOVA $(\mathrm{P}>0,05)$ e ${ }^{2}$ Kruskal Wallis $(\mathrm{P}>0,05)$. Treze animais não apresentaram CL no momento da avaliação (cinco do T1, quatro do T2, quatro do T3).

Não houve diferença $(\mathrm{P}>0,05)$ (Tab. 2) para a taxa de gestação observada no presente trabalho, sendo obtidos valores de $40 \% ; 24 \% ; 36 \%$, para $\mathrm{T} 1, \mathrm{~T} 2$ e $\mathrm{T} 3$, respectivamente. Esse resultado diverge do encontrado por Tortorelle et al. (2013), que, após a administração de eCG, obtiveram maior a taxa de gestação no grupo com aplicação da eCG $48 \mathrm{~h}$ antes da retirada do implante $(27,27 \%)$ do que no grupo que aplicou a eCG no momento da retirada $(16,0 \%)$.

A antecipação da aplicação da eCG não melhorou os índices de crescimento folicular e de ovulação, o tamanho do CL formado e a taxa de prenhez em vacas de leite submetidas a protocolos de sincronização de ovulação em programas de IATF, sendo, então, recomendada a utilização da eCG no momento da retirada do implante, por uma questão de otimização do manejo.

\section{REFERÊNCIAS}

ALI, A. Effect of time of eCG administration on follicular response and reproductive performance of FGA-treated Ossime ewes. Small Ruminant Res., v.72, p.33-37, 2007.
BARUSELLI, P.S.; REIS, E.L.; MARQUES, M.O. et al. The use of hormonal treatments to improve reproductive performance of anestrous beef cattle in tropical climates. Anim. Reprod. Sci., v.82, p.479-486, 2004.

BISTER, J.L.; NOEL, B.; PERRAD, B. et al. Control of ovarian follicles activity in the ewe. Domest. Anim. Endocrinol. v.17, p.315-328, 1999.

EDMONSON, A.J.; LEAN, I.J.; WEAVER, L.D. et al. A body condition scoring chart for Holstein dairy cows. J. Dairy Sci. v.72, p.68-78, 1989.

ESTAÇÕES concencionais. Brasília: INMET, 2015. Disponível em: <http://www.inmet.gov.br/ portal/index.php?r=home/page\&page=rede_estac oes_conv_graf >. Acesso em: 2 maio 2016.

GIMENES, L.U.; CARVALHO, N.A.T.; SÁ FILHO, M.F. et al. Capacidade ovulatória em novilhas Bos indicus. Acta Sci. Vet., v.33, p.209, 2008.

KIYA, C.K.; BISCARDE, C.E.A.; SILVA, L.F.M.C. et al. Diferentes momentos de administração de eCG associada ou não à hCG em protocolo curto para IATF em ovelhas deslanadas do semiárido. Magistra, v.26, p.535542, 2014. 
MANDIKI, S.N.; NOEL, B.; BISTER, J.L. et al. Pre-ovulatory follicular characteristics and ovulation rates in different breed crosses, carriers or non-carriers of the Booroola or Cambridge fecundity gene. Anim. Reprod. Sci, v.63, p.77-88, 2000.

MANN, G.E. Corpus luteum size and plasma progesterone concen- tration in cows. Anim. Reprod. Sci., v.115, p.296-299, 2009.

MARQUES, M.O.; REIS, E.L.; CAMPOS FILHO, E.P.; BARUSELLI, P.S. Efeitos da administração de eCG e de Benzoato de Estradiol para sincronização da ovulação em vacas zebuínas no período pós-parto. In: SIMPOSIO INTERNACIONAL DE REPRODUCCIÓN ANIMAL, 5., 2003, Cordoba. Anais... Cordoba: [s.n.], 2003. p.27-29.

MURPHY, B.D.; MARTINUK, S.D. Equine chorionic gonadotropin. Endocrinol. Rev., v.12, p.27-44, 1991.

NEVES, M.M.; MARQUES JÚNIOR, A.P. Proporção volumétrica dos constituintes do corpo lúteo de Nelore. Arq. Bras. Med. Vet. Zootec., v.58, p.944-946, 2006.

PIERSON, R.A.; GINTHER, O.J. Ultrasonic imaging of the ovaries and uterus in cattle. Theriogenology, v.29, p.21-37, 1988.

PINHEIRO, O.L.; BARROS, C.M.; FIGUEREDO, R.A. et al. Estrus behavior and the estrus-to-ovulation interval in Nelore cattle (Bos indicus) with natural estrus or estrus induced with prostaglandin F2 or norgestomet and estradiol valerate. Theriogenology, v.49, p.667-681, 1998.
SARTORI, R.; ROSA, G.J.M.; WILTBANK, M.C. Ovarian structures and circu- lating steroids in heifers and lactating cows in summer and lactating and dry cows in winter. J. Dairy Sci., v.85, p.813-822, 2002.

SIROIS, J.; FORTUNE, J.E. Ovarian follicular dynamics during the estrus cycle in heifers monitored by real-time ultrasonography. Biol. Reprod., v.39, p.308-317, 1988.

SOUMANO, K.; LUSSIER, J.G.; PRICE. C.A. Levels of messenger RNA encoding ovarian receptors for FSH and $\mathrm{LH}$ in cattle during superovulation with equine chorionic gonadotrophin versus FSH. J. Endocrinol., v.156, p.373-378, 1998.

TABELA 4 - Efetivo dos rebanhos de médio porte em 31.12, segundo as grandes regiões e as Unidades da Federação - 2013. [Rio de Janeiro]: IBGE, 2013. Disponível em: <ftp://ftp.ibge.gov.br/Producao_Pecuaria/Produc ao_da_Pecuaria_Municipal/2013/tabelas_pdf/tab 04.pdf $>$. Acesso em: 9 de abril de 2016.

TORTORELLA, R.D.; FERREIRA, R.; SANTOS, J.T. et al. The effect of equine chorionic gonadotropin on follicular size, luteal volume, circulating progesterone concentrations, and pregnancy rates in anestrous beef cows treated with a novel fixed-time artificial insemination protocol. Theriogenology, v.79, p.204-209, 2013.

VIANA, J.H.; FERREIRA, A.M.; SÁ, W.F.; CAMARGO, L.S. Características morfológicas e funcionais do corpo lúteo durante o ciclo estral em vacas da raça Gir. Arq. Bras. Med. Vet. Zootec, v.51, p.251-256, 1999.

VISHWANATH, R. Artificial insemination: the state of the art. Theriogenology, v.59, p71-84, 2003. 